\title{
Ecología-mundo, un nuevo paradigma para el estudio de las migraciones internacionales ${ }^{1}$
}

\author{
World-ecology, a new paradigm for the study of \\ international migrations
}

\author{
Yoan Molinero Gerbeau \\ Investigador Consejo Superior de Investigaciones Científicas \\ yoan.molinero@cchs.csic.es (ESPAÑA) \\ Gennaro Avallone \\ Investigador Universidad de Salerno (ITALIA)
}

Recibido: 24.102018

Aceptado: 03.12.2019

\section{RESUMEN}

A lo largo del siglo XX y comienzos del XXI la comunidad académica ha desarrollado numerosas teorías para explicar el fenómeno de las migraciones internacionales, tal como Massey et al. (1998) recogieron en su famoso libro "Worlds in motion". Sin embargo, una mayoría de estudios producidos en este campo se han referido principalmente a casos empíricos recurriendo al uso de los principales paradigmas cuyas explicaciones de la realidad han tendido a reiterarse. Rompiendo con esta tendencia, en los últimos años han ido desarrollándose nuevos enfoques, cuyo carácter interdisciplinar ha permitido llevar a cabo estudios novedosos que han aportado respuestas diferentes a los interrogantes más clásicos acerca de las migraciones internacionales.

Esta investigación pretende contribuir a dicha tendencia presentando un nuevo paradigma proveniente de las Relaciones Internacionales: la ecologíamundo. Situando las relaciones socio-ecológicas como epicentro del desarrollo del capitalismo global, el paradigma creado por Jason W. Moore permitirá no solo llevar cabo novedosas investigaciones de los fenómenos sociales y políticos, sino que abrirá una nueva ventana teórica para conectar sus postulados con

${ }^{1}$ Esta investigación ha sido financiada por la II Convocatoria de Contratos de Investigación "Ford España - Apadrina la Ciencia" con el apoyo del Ford Motor Company Fund 
una infinidad de áreas de estudio. En base a su perspectiva holística, histórica y transnacional, la ecología-mundo se ofrece como un nuevo paradigma idóneo para explicar fenómenos globales tan diversos como el cambio climático, la crisis económica o la movilidad humana. Gracias a sus múltiples herramientas teóricas, el paradigma aquí presentado ofrece una infinidad de posibilidades para el desarrollo de estudios críticos que expliquen las migraciones humanas desde prismas hasta ahora inexplorados. El objetivo de este artículo por lo tanto será doble. En primer lugar, se perseguirá caracterizar la ecología-mundo definiendo sus raíces teóricas y sus principales planteamientos analíticos con el objetivo de dar a conocer una propuesta hasta ahora largamente desconocida en el mundo hispanoparlante. En segundo lugar, se buscará resaltar las principales contribuciones que los constructos teóricos del paradigma ofrecen para el estudio de las migraciones.

\section{PALABRAS CLAVE}

Ecología-mundo, migraciones internacionales, cadenas globales, estructuralismo, capitalismo.

\section{ABSTRACT}

Throughout the twentieth and early twenty-first centuries, the academic community has developed numerous theories to explain the phenomenon of international migration, as Massey et al. (1998) collected in his famous book «Worlds in motion». However, a majority of studies produced in this field have referred mainly to empirical cases resorting to the use of the main paradigms whose explanations of reality have tended to be reiterated. Breaking with this trend, in recent years, new approaches have been developed, whose interdisciplinary character has allowed to carry out innovative studies that have provided different answers to the most classic questions about international migrations.

This research aims to contribute to this trend by presenting a new paradigm from International Relations: the world-ecology. Placing socio-ecological relations as the epicenter of the development of global capitalism, the paradigm created by Jason W. Moore will allow not only to carry out novel investigations of social and political phenomena, but will also open a new theoretical window to connect his postulates with an infinity of study areas. Based on its holistic, historical and transnational perspective, world-ecology offers a new paradigm suitable to explain diverse global phenomena such as climate change, economic crisis or human mobility. Thanks to its multiple theoretical tools, the paradigm presented here offers an infinity of possibilities for the development of critical studies that explain human migrations from prisms until now unexplored. The objective of this article will therefore be twofold. Firstly, it will define the worldecology paradigm by identifying its theoretical roots and its main analytical 
approaches. Secondly, it will seek to highlight the main contributions that the theoretical constructs of the paradigm offer for the study of migrations.

\section{KEY WORDS} talism

World-ecology, international migrations, global chains, structuralism, capi-

\section{INTRODUCCIÓN}

El ámbito de estudio de las migraciones internacionales ha sido tradicionalmente un campo en disputa entre corrientes diversas y contrapuestas cuyo intento por explicar las dinámicas que inciden en la movilidad humana ha partido desde los más diversos presupuestos. Quizás la obra que mejor haya reflejado y categorizado este pluralismo teórico fue el pionero libro "Worlds in Motion. Understanding International Migration at the End of the Millennium" coordinado por Massey et al. (1998) que dio cuenta tanto de aquellas corrientes que habían hegemonizado el análisis de las migraciones a lo largo del siglo XX, como de un número de incipientes teorías que pretendían cuestionar sus planteamientos. Desde entonces, nuevas teorías han ido surgiendo y de manera habitual, nuevos estudios han tratado de compilar esta diversidad, mostrando que el campo de los estudios migratorios no solo es dinámico, sino que es de rabiosa actualidad (King 2012).

Lamentablemente, y pese al carácter pluridisciplinar del propio objeto de estudio en cuestión, las corrientes académicas han tendido a ser compartimentos estancos donde autores encuadrables en una determinada escuela han seguido una línea de estudios marcada o donde nuevas teorías han permitido entender nuevos fenómenos, pero siempre apoyadas en esquemas clásicos. El objetivo de este artículo es el de presentar un paradigma nuevo, conocido como "ecologíamundo", poco explorado en los estudios migratorios, pero cuya utilidad para el análisis de este fenómeno es indudable dado su enfoque holístico y su aportación de innovadoras herramientas teóricas.

Tomando como base el marco del sistema-mundo, la fractura metabólica y la reproducción social, la ecología-mundo incidirá con fuerza en el campo de las Relaciones Internacionales permitiendo una reinterpretación total de los procesos globales. Así pues, se trata de una corriente que no nace del mundo de los estudios migratorios, sino que surge como un marco de estudio de la realidad internacional que permitirá explicar desde un punto de vista Estructuralista ${ }^{2}$

${ }^{2}$ Es tradición de la disciplina de Relaciones Internacionales escribir con mayúscula tanto las referencias a ésta como los nombres de los paradigmas que la componen para así evitar que estos términos, de carácter académico, se confundan con su sentido literal. Tal como señalan Brown y Ainley "Relaciones Internacionales es el estudio de las relaciones internacionales" (2011: 1) mostrando cómo el uso de la mayúscula impide confundir los significados de términos idénticos. Por 
fenómenos como éste. Precisamente por ello, es decir, por su carácter exógeno a los estudios migratorios, la ecología-mundo ofrece la oportunidad de abrir una nueva ventana teórica y así aportar nuevas categorías y conceptos que enriquezcan el análisis en este campo de estudio.

El objetivo de este artículo por lo tanto será doble. Por un lado, se perseguirá presentar una compleja teoría, largamente desconocida en el mundo hispano parlante, y por el otro, se buscará resaltar la utilidad de este marco teórico para el estudio de las migraciones internacionales.

Para ello el artículo se estructurará de la siguiente manera. Primeramente, se analizarán los precedentes teóricos de los que se ha alimentado la ecologíamundo con el fin de entender su procedencia y sus fuentes teóricas. En una segunda instancia se tratará de caracterizar sus principales rasgos definitorios para posteriormente resaltar, en una tercera sección, los aportes más sustanciales que puede hacer la ecología-mundo al campo de los estudios migratorios. El artículo cerrará finalmente con las conclusiones.

\section{FUNDAMENTOS DE LA ECOLOGÍA-MUNDO}

\subsection{De la economía-mundo al sistema-mundo. La evolución de la escuela Estructuralista de Relaciones Internacionales}

El paradigma de la ecología-mundo irrumpió con fuerza en el estudio de las Relaciones Internacionales principalmente en 2015 cuando su acuñador, Jason W. Moore, investigador del Centro de Estudios Fernand Braudel (Universidad de Binghamton, Nueva York), publicó su libro "Capitalism in the Web of Life: Ecology and the Accumulation of Capital", un manuscrito que condensará de manera sistematizada los cimientos de su teoría, alcanzando así un público mucho mayor que sus precedentes escritos ${ }^{3}$. Sin embargo, la construcción teórica de este paradigma data de ocho años antes, pues la primera mención al sistema capitalista global como una ecología-mundo puede encontrarse en su artículo "Capitalism as World-Ecology: Braudel and Marx on Environmental History" (Moore 2003).

La ecología-mundo se encuadra dentro de la tradición Estructuralista de Relaciones Internacionales, un conjunto de teorías que emergieron en los años 50 con el objetivo de romper con la hegemonía que hasta entonces habían tenido las escuelas Realistas y Liberales en la construcción de esta disciplina (Brown

\footnotetext{
ello, en este texto se empleará la mayúscula para hacer referencia a los diversos paradigmas de la disciplina tales como el Realismo, el Estructuralismo o el Liberalismo.

${ }_{3}^{3}$ Prueba de ello son las 731 citas que acumulaba este libro en poco más de tres años en Google Scholar a fecha de agosto de 2018.

Véase https://scholar.google.es/citations?user=IWNMcvQAAAAJ\&hl=es

${ }^{4}$ En realidad, el primero en emplear el término "ecología-mundo" fue Wallerstein (1974) aunque no desarrolló teóricamente el concepto sino que lo utilizó de forma ocasional para referirse ya a la estrecha vinculación entre capitalismo y naturaleza.
} 
y Ainley 2009). Para los teóricos Estructuralistas, la unidad de análisis no era el Estado sino la superestructura del sistema capitalista global donde Norte y Sur, o más bien, centro y periferia, cumplen funciones diferenciadas en una misma relación sistémica. Con el telón de fondo de la Guerra Fría y la descolonización, el Estructuralismo perseguía revelar la existente jerarquía global entre áreas del mundo no solo para denunciar la desigualdad del sistema global, sino para orientar cambios estructurales en favor de la periferia. Con teóricos como Raúl Prebisch a la cabeza, el Estructuralismo acuñó importantes desarrollos teóricos tales como la Teoría de la Dependencia, cuyos efectos se plasmaron en ciertas políticas impulsadas por determinados Estados No Alineados, como fueron, por ejemplo, los planes de Industrialización por Sustitución de Importaciones (Caldentey et al. 2012).

Sin embargo, esta corriente no estará exenta de críticas, siendo muy comunes aquellas que acusaban a sus exponentes de ser más idealistas que científicos (Warren 1980) o de exponer un reformismo limitado (Street y James 1980). Así, en los 70, surge una nueva ola de Estructuralistas amparados por su rechazo al auge del neoliberalismo y la expansión de los Planes de Ajuste Estructural implementados por el FMI y el Banco Mundial en las áreas periféricas del mundo. Este nuevo Estructuralismo tomará como referencia fundamental las leyes sobre el capital de Karl Marx y el análisis geo-histórico de fases de acumulación de Fernand Braudel, adquiriendo de esta manera un carácter más marcado de economía política. El principal referente de esta oleada será Immanuel Wallerstein, cuya trilogía sobre "El moderno sistema mundial" (ampliada a tetralogía en 2011) se convertirá en el principal referente del Estructuralismo al situar su teoría del sistema-mundo (Wallerstein 1974) como la columna vertebral de esta escuela de pensamiento.

Como su propio nombre sugiere, la perspectiva de la ecología-mundo, bebe profundamente de las teorías del sistema-mundo wallerstenianas, que a su vez partieron del concepto de economía-mundo iniciado por Braudel (Wallerstein 2004). La etiqueta "-mundo" compartida por las tres corrientes muestra no solo la conexión teórica existente entre ellas, sino la pretensión que primero Wallerstein (1974) y luego Moore (2003) han tenido de auparse a una tradición a la que indisimuladamente han querido superar aportando un marco más amplio o más complejo.

Braudel inició esta tradición con su pionero estudio sobre el mundo Mediterráneo en el siglo XVI (1976). Para el historiador francés, esta época marcó un cambio fundamental en el devenir de las Relaciones Internacionales dado que las incipientes relaciones económicas de tipo capitalista en la región mostraban el nacimiento de un sistema que superaba el marco estatal. La unidad de análisis aquí era la "economía-mundo", siendo esta una "amplia zona geográfica entre la que hubo una división del trabajo y por lo tanto un significativo intercambio interno de bienes básicos o esenciales, así como de flujos de trabajo y capital" (Wallerstein 2004: 23). La economía-mundo, por lo tanto, se caracterizará por no disponer de una unidad política, sino por ser un sistema de acumulación capi- 
talista dentro del cual las diferentes áreas en relación jugarán un rol estructural, funcional y diferenciado para el sistema.

Wallerstein otorgará validez al análisis braudeliano, pero considerará que el fenómeno del surgimiento del sistema capitalista, al ser estudiado desde una perspectiva histórica más amplia, revelará una tendencia a convertirse en un sistema global, es decir en un sistema-mundo. Así pues, el desarrollo de la economía-mundo mediterránea en el siglo XVI, no sería más que la génesis de un modelo económico que a diferencia de los sistemas previos, se caracterizará por perseguir la acumulación infinita de capital como razón de ser (Wallerstein 2000b). La propia esencia del capitalismo, por lo tanto, llevará al sistema a buscar una expansión constante, tanto productiva como geográfica, en un proceso que, siguiendo el análisis de Wallerstein, lo llevará a convertirse en el sistema global que regirá las Relaciones Internacionales desde su puesta en marcha hasta la actualidad. De esta manera, las diferentes economías-mundo de las fases iniciales, para Wallerstein, serán tan solo embriones de lo que después será el sistema-mundo, una vez se unan o sean superadas integrándose en una única área más amplia.

Siguiendo esta premisa, Wallerstein definirá el sistema-mundo como "una zona espacio-temporal que atraviesa múltiples unidades políticas y culturales representando una zona integrada de actividad e instituciones que obedecen ciertas normas sistémicas" Wallerstein 2004: 17). Se trata por lo tanto de un sistema global, en el que los diferentes actores, ya sean Estados, mercados o empresas transnacionales (entre muchos otros) ocupan un determinado espacio funcional a la estructura capitalista total.

El sistema-mundo capitalista se estructurará en zonas de poder, existiendo un centro -con una alta tasa de concentración de capital fijo, especializado en la producción de productos manufacturados- y una periferia -caracterizada por una alta tasa de capital variable, principalmente insumos de producción como materias primas, pero también de un importante ejército de reserva de trabajadores-. Wallerstein, además, mencionará la existencia de áreas semi-periféricas (donde existen parte de ambos procesos) y una "arena exterior", siendo espacios del globo no integrados en la estructura del sistema-mundo.

La división centro-periferia será una dimensión crucial en la teoría wallersteniana pues será integrada por la mayoría de los teóricos Estructuralistas, pero también por una importante parte de las corrientes críticas de Relaciones Internacionales, Ciencia Política y Sociología. Esta visión permitirá cuestionar doctrinas impuestas por el realismo tales como la corriente desarrollista y su división entre "Estados desarrollados" y "Estados en vías de desarrollo", o perspectivas más geopolíticas como las que hablan de un Norte y un Sur globales, así como el discurso sobre el "Tercer Mundo", cuyo origen, en este caso, es Estructuralista La perspectiva centro-periferia cuestionaba por tanto perspectivas teleológicas

${ }^{5}$ Cabe señalar que si bien la visión centro-periferia es "canónica" dentro de la teoría del sistema-mundo, algunos de sus teóricos han dado por hecho que Norte y Sur son sinónimos de la misma (Arrighi 
sobre el supuesto avance de unas áreas del mundo sobre otras en un eje temporal hacia el desarrollo, o que la existencia de bloques o posiciones geográficas determinasen la condición de los Estados en el plano geopolítico global. Para el sistema-mundo, el sistema mundial es el capitalismo, y el poder ejercido por los Estados, pero también por los demás actores, dependía esencialmente de la función sistémica que cumplían en dicha superestructura.

Sintetizar el resto de contribuciones teóricas al análisis internacional que aportó esta perspectiva es un arduo trabajo, pero no podrían dejar de mencionarse dos conceptos fundamentales que contribuyeron decisivamente al armazón teórico del sistema-mundo: las fases de acumulación y las cadenas globales.

\subsubsection{Fases de acumulación}

Aunque el concepto de fases de acumulación como tal está fuertemente presente en la obra de Immanuel Wallerstein (2000a), su germen puede encontrarse en el concepto de "coyunturas" usado por Braudel (1976) que se apoya en las tesis de "El Capital" de Marx. Sin embargo, quién más ahondará en su estudio será Giovanni Arrighi cuya extensa obra contribuirá decisivamente a reforzar el análisis de las coyunturas económicas en el sistema-mundo. Curiosamente, uno de los manuscritos donde mejor quedará sintetizado este concepto será titulado "Capitalist Development in World-Historical Perspective" y será escrito en coautoría con Jason W. Moore antes de que éste desarrollara la perspectiva de la ecología-mundo (Arrighi y Moore 2001).

Las fases de acumulación son una división de la historia del capitalismo en periodos históricos globales. Según Arrighi y Moore (2001), éste habría vivido cuatro grandes ciclos históricos: el Genovés-Ibérico del siglo XV al XVII, el Holandés del siglo XVI al XVIII, el Británico desde la mitad del XVIII al XX y el Estadounidense del XIX hasta la actualidad. Estos ciclos seguirían la fórmula del capital de Marx donde habría habido dos fases de acumulación diferenciadas en las que una primera expansión material sería después seguida de una expansión financiera. La expansión material se da cuando agencias líderes del centro del sistema-mundo han sido capaces de impulsar al sistema hacia más amplias y profundas divisiones del trabajo generando mayores retornos al capital invertido. La segunda expansión, de tipo financiero, es la primera señal de crisis de un ciclo de acumulación, pues significa que la agencia líder no es capaz de expandir más el modelo productivo mediante la producción y el comercio, pasando a extraer beneficios de la especulación e inversión financieras.

Gracias a la perspectiva de los ciclos de acumulación, es posible identificar quién es la agencia líder en cada fase del capitalismo, cuál es el modelo productivo imperante y en qué estado se encuentra el capitalismo global (a modo de poder intuir cambios estructurales). De esta manera, el hecho de que el neoliberalismo sea una expansión de tipo financiero es interpretado por los Estructuralistas actuales como una señal de agotamiento de ciclo (Arrighi y Moore 2001) cuya crisis, iniciada en 2003 pero manifestada de forma contundente en 2008, 
podría dar pie tanto a una nueva fase de acumulación como al agotamiento del propio sistema (Moore 2015b).

\subsubsection{Cadenas Globales}

El concepto de cadenas globales es quizás uno de los más universales de la teoría del sistema-mundo, pues ha sobrepasado su propio marco teórico, siendo comúnmente empleado por economistas e investigadores no necesariamente afines a esta teoría (de Castro 2018). Fue acuñado por Terence Hopkins y Wallerstein en su famoso artículo "Commodity Chains in the World-Economy prior to 1800" (1986) donde definieron estas cadenas como "redes de procesos productivos y laborales cuyo resultado final es una mercancía terminada" (Hopkins y Wallerstein 1986: 159). Desde entonces la perspectiva de las cadenas globales ha crecido exponencialmente, existiendo varias escuelas y corrientes teóricas al respecto (Bair 2009).

La riqueza teórica existente en el campo de estudio de las cadenas dificulta el poder definir sus características esenciales, pues existen tendencias de estudio enfrentadas tales como la perspectiva de las "Cadenas Globales de Mercancías", las "Cadenas Globales de Valor", las "Redes Globales de Producción" o los "Estudios Regionales del Desarrollo" (de Castro, 2018). Sin embargo, todas coinciden en partir del hecho de que se trata de una metáfora que permite mostrar cómo "los procesos de trabajo y de producción transnacional conectan materialmente las economías, las empresas, los trabajadores y los hogares en la economía global contemporánea" (Mezzadra y Neilson 2017: 145).

$\mathrm{Al}$ concebir los procesos productivos globales como cadenas se hace, por un lado, alusión a la línea de ensamblaje fordista cuya ideología ha configurado el modelo productivo hegemónico del sistema-mundo, y, por el otro, sugiere el símil, siguiendo una retórica marxista, que presenta las cadenas como grilletes que atan a los trabajadores al capital.

Al componerse de eslabones, la cadena permite entender cómo algunos procesos productivos se localizan en el centro y otros en la periferia en un proceso que es mutable y líquido a través del tiempo y el espacio (Bair 2009). Así, se entienden mejor los procesos de dispersión geográfica de la producción capitalista pudiéndose arrojar luz sobre las estructuras de gobernanza que determinan la distribución de los recursos materiales, financieros y humanos a nivel global (Mezzadra y Neilson 2017). En lo que respecta al eslabón del trabajo, esta perspectiva permite identificar la procedencia y movilización del ejército de reserva de trabajadores, pero también contribuye a poder identificar las luchas y resistencias que se dan en el mismo, señalando el carácter político de su configuración (de Castro 2018) y otorgando espacios para el estudio de la agencia de los protagonistas (Molinero 2018). 


\subsection{Ecología-mundo, un nuevo marco interpretativo}

\subsubsection{Dualismo cartesiano y centralidad de la naturaleza}

Como el propio Wallerstein explicó (2000b), el paradigma del sistema-mundo fue concebido como una teoría viva, en constante construcción, y en permanente diálogo con la comunidad científica. No se trataba de un armazón teórico que pudiera aportar todas las respuestas a la compleja realidad internacional, sino que se trataba más bien de un marco interpretativo que permitía entender los procesos transnacionales impulsados por la estructura del sistema capitalista global. La notoriedad alcanzada por esta teoría provocó que fuera "alabada, atacada y muchas veces infrarrepresentada o malinterpretada" (Wallerstein 2000b: xi) pero también discutida por quiénes aceptaban el marco general, pero consideraban que debía avanzar en una u otra dirección.

La ecología-mundo nacerá en el marco de una crítica constructiva al paradigma elaborado mayormente por Wallerstein. Para Jason W. Moore, el sistemamundo no era una teoría equivocada, pero sí presentaba, a juicio de este autor, importantes lagunas que debían ser superadas. Si bien Moore tomaba por válido el esquema centro-periferia y los demás elementos definitorios de la teoría, como las fases de acumulación o las cadenas globales, consideraba que tanto Braudel como Wallerstein habían pasado muy por encima de un elemento determinante: la relación del capitalismo con la naturaleza (Moore 2003).

Para Jason W. Moore, las ciencias modernas están aquejadas de un mal que denominó como "dualismo cartesiano" (Moore 2015a), esta es una narrativa según la cual sociedad y naturaleza son dos entes no solo diferenciados, sino opuestos. Si la sociedad representa la civilización y el progreso, el mundo de la naturaleza es el mundo de la barbarie, de lo salvaje, por lo que las ciencias modernas se construyeron en torno a un consenso ante el cual la sociedad podía ejercer un control deliberado sobre la naturaleza. Sin embargo, para Moore, esta artificial división constituye una falacia diseñada con el objetivo de permitir un ejercicio del poder indispensable para el proceso de acumulación (Moore 2017a). El capitalismo, al construir la naturaleza como aquello que está fuera de la civilización, es decir, como un conjunto de valores pre-fabricados disponibles de forma gratuita para su apropiación, estructura una racionalidad según la cual ésta es directamente apropiable para la producción. De esta misma forma, el dualismo cartesiano organizó el pensamiento europeo para concebir una separación total entre campo y ciudad, o lo que es lo mismo, generar una fractura metabólica, estableciendo una jerarquía según la cual, la primera debía estar al servicio de la segunda pues el campo significa "atraso" y la ciudad "progreso" (Moore 2003).

El dualismo cartesiano constituye un a priori de las ciencias modernas, estando tan establecido en el inconsciente de quienes hacen estudios sociales, que se ha instalado como un consenso incuestionado, de ahí que la consideración de la naturaleza como un objeto externo y apropiable permeara también al Estructuralismo. Esto no significa que los autores fundamentales de la corriente negaran la importancia de la naturaleza en sus análisis, pues según Moore, las bases de 
la ecología-mundo pueden encontrarse ya en las obras de Braudel, Wallerstein y Marx. Todos en mayor o menor medida señalaron en algún punto de sus escritos que la naturaleza jugó un papel determinante en el desarrollo del capitalismo, pero a juicio de Moore, prestaron una mayor atención al trabajo y su relación con el capital que a dicha dimensión (2003).

La ecología-mundo parte de la premisa de que la naturaleza es un elemento determinante para comprender la evolución del sistema-mundo. Frente a aquellos discursos cartesianos que, por ejemplo, señalan los desastres ecológicos del capitalismo como si fueran una consecuencia de sus acciones, la ecología-mundo propone una visión diferente: el capitalismo es un proyecto socio-ecológico que persigue la acumulación infinita movilizando y apropiándose de las naturalezas humanas y extra-humanas (Moore 2017a). La ecología-mundo, por tanto, entiende que la naturaleza no es un ente diferenciado de los humanos, sino que ambos forman una única matriz, siendo explotados de forma sistémica por el capitalismo global.

"El capitalismo no es solo parte de una ecología, sino que es una ecología - un conjunto de relaciones integrando poder, capital y naturaleza. Por lo tanto, cuando escribimos - y añadimos el guion- ecología-mundo, nos basamos en viejas tradiciones de "sistemas-mundo" para decir que el capitalismo crea una ecología que se expande a lo largo del planeta a través de fronteras, impulsado por fuerzas de acumulación infinita" (Patel y Moore 2017: 38).

Este enfoque, por lo tanto, no se refiere a la ecología del mundo, sino que define al capitalismo como un proyecto ecológico, un sistema-mundo que coproduce la naturaleza (tanto humana como extrahumana) poniéndola al servicio de la acumulación. Así, para Moore, el capitalismo más que un sistema (un sistema-mundo) es una ecología (una ecología-mundo), "una civilización diferenciada y unificada a través de la cual la acumulación de capital, la producción de la naturaleza, y la búsqueda del poder forman una totalidad histórico-mundial" (Moore 2013: 16).

El capitalismo, con su surgimiento, impuso una nueva forma de organización de la naturaleza en un proceso de apropiación que ilustran perfectamente dos conceptos clave de la ecología-mundo: los cuatro factores baratos y las fronteras de producción.

\subsubsection{Los cuatro factores baratos y las fronteras de producción}

El concepto de "barato" estará muy presente a lo largo de la obra de Jason W. Moore (2016a) hasta el punto de que su último libro hasta la fecha, escrito con Raj Patel, será un análisis histórico del crecimiento capitalista a través de "siete cosas baratas" (Patel y Moore 2017). Lo barato, o el abaratamiento, para la ecología-mundo, es un concepto sistémico pues tiene que ver con el conjunto de estrategias que desarrolla el sistema capitalista para maximizar la acumulación. El análisis de la ecología-mundo reflejará que cada fase de acumulación del 
capitalismo (o cada régimen ecológico) tendrá que ver con la apropiación a gran escala de naturalezas baratas (Moore 2015b). En concreto, Moore identificará cuatro factores esenciales para garantizar una fase expansiva de acumulación: el trabajo, la comida, las materias primas y la energía. Estos cuatro elementos serán los que Moore denomine como los "cuatro factores baratos" (Moore 2015a).

La ecología-mundo demostrará que cada una de las cuatro fases históricas de acumulación han podido producirse gracias a que el sistema capitalista ha sido capaz de obtener y reproducir los cuatro factores a bajo coste o incluso a coste nulo (este último siendo la estrategia ideal perseguida por el sistema). Las fases expansivas, por lo tanto, se han debido al hecho de que trabajo, comida, materias primas y energía han podido ser obtenidos a nivel global de forma barata gracias a los modelos productivos imperantes en cada época (Moore 2015a).

Cuando Moore habla de trabajo se refiere a la mano de obra proletaria, cuyo rol central en el proceso productivo es innegable. La comida es otro de los factores clave, pues de su accesibilidad depende la reproducción de la mano de obra y de su bajo coste depende que los bajos salarios de los proletarios sean suficientes para su adquisición. Las materias primas y la energía serán los otros dos insumos fundamentales del proceso productivo. Estos cuatro elementos están estrechamente vinculados entre sí y forman un equilibrio indispensable para garantizar la acumulación.

Por ejemplo, un aumento del precio de la comida implicará necesariamente un aumento del coste del trabajo pues, para acceder a esta y garantizar la supervivencia de la mano de obra, los salarios deberán aumentar en consonancia. De igual manera, los aumentos en el coste de las materias primas o la energía provocarán una carestía de la producción que implicará caídas. La centralidad de estos elementos muestra la interconexión entre las naturalezas humanas y extra-humanas pues su explotación conjunta es indispensable para mantener la acumulación (Moore 2015a). Así, se puede ver que el capitalismo más que un sistema-mundo es una ecología-mundo: una forma de producir, reproducir y explotar las naturalezas mundiales.

Históricamente, cada fase de acumulación ha sido posible por la obtención de los cuatro factores baratos, pero los modelos nunca han sido infinitos, pues el capitalismo, en su afán acumulativo, agota las fuentes y los recursos, estando abocado a una crisis permanente (Moore 2015b). Cuando uno o varios de los cuatro factores no pueden ser obtenidos de forma gratuita o barata, el sistema entra en crisis, debiendo expandirse geográficamente, desarrollar nuevas técnicas productivas o cambiar los modelos de producción para poder reactivar su obtención a bajo coste. Dicha reactivación pasa por la apropiación de nuevas fronteras de producción.

El concepto de frontera de producción es clave para comprender las bases de la ecología-mundo, pues permite entender los cambios tanto sistémicos como de fases de acumulación. La frontera de producción muestra el límite de un determinando momento ecológico, por ejemplo, cuando ya el sistema, con su actual configuración no es capaz de obtener uno o varios de los cuatro factores a bajo coste. La crisis generada por esto empujará al sistema a avanzar hacia otro 
modelo, a apropiarse de una nueva frontera de producción, que permita reactivar la acumulación. A nivel histórico ha habido numerosas fronteras de producción que han pasado desde la expansión geográfica, por ejemplo, con el colonialismo, al cambio de modelos productivos, como la conversión al modelo de producción industrial que convirtió al tercer estado en proletariado (Patel y Moore 2017). Todos estos cambios son nuevas fronteras de producción de las que el sistema se ha apropiado tras haber agotado su anterior fase. Por poner un ejemplo más directo, se puede hablar del trabajo esclavo en las colonias, cuya apropiación gratuita permitió la expansión de la ecología-mundo a América. En cambio, cuando este ya no era apropiable, por las resistencias de los propios esclavos, las dificultades crecientes para su obtención en África o por las leyes de prohibición, la ecología-mundo tuvo que reconfigurarse y proletarizar a las poblaciones autóctonas de los imperios para garantizar, a nivel sistémico, el trabajo barato (Moore 2015a).

Los cuatro factores baratos y las fronteras de producción muestran claramente tanto la intersección que el paradigma de la ecología-mundo tiene con el sistema-mundo como la configuración de un marco teórico marcadamente diferenciado de su predecesor.

\subsubsection{Otros desarrollos teóricos de la ecología-mundo}

Si bien se han definido en las secciones anteriores los rasgos más esenciales de la teoría de la ecología-mundo, el paradigma se ha ampliado notablemente, dando pie a una serie de nuevas líneas de investigación que han extendido su campo de estudio. Cobijados en su mayoría (aunque no exclusivamente) bajo el paraguas del "World-ecology Research Network" coordinado por Jason W. Moore, numerosos estudios han ido aplicando este paradigma como marco teórico.

Principalmente, quiénes han contribuido a desarrollar la teoría han realizado estudios agrarios, historiográficos, medioambientales, sobre extractivismo, acaparamiento de tierras, ecología política, globalización etc.

A parte de estos, cabe destacar tanto la importante dialéctica mantenida por los teóricos de la ecología-mundo con quiénes sostienen la teoría de la fractura metabólica (Moore 2017b), como el profundo cuestionamiento que han realizado al concepto de Antropoceno. Moore y sus colaboradores señalaron que este último sugería que la humanidad era la causante del cambio climático cuando en realidad quién ha acelerado el fin de nuestra era climática es el sistema capitalista, es decir la ecología-mundo, pudiéndose definir de forma más concreta que vivimos no en la era del Antropoceno, sino del Capitaloceno (Moore 2016a).

Aunque ya ha sido mencionado previamente, Moore (2015b) en sus estudios también ha incidido mucho en el análisis de la actual crisis global iniciada en

\footnotetext{
${ }^{6}$ Véase https://worldecologynetwork.wordpress.com/
} 
2003, conjeturando sobre si el sistema se enfrenta a un nuevo fin de ciclo de acumulación, o a su crisis terminal.

Por último, y para cerrar este breve apartado, no debería dejar de mencionarse la fuerte inspiración que las teorías feministas han aportado al paradigma de la ecología-mundo. El nexo sistémico entre el trabajo femenino no pagado y el desarrollo capitalista es central para esta perspectiva, donde la teoría de la reproducción social (sobre todo por la conexión que realiza entre comida y trabajos baratos como indispensables para la reproducción del proletariado global) ha jugado un rol fundamental en su desarrollo teórico.

\section{ECOLOGÍA-MUNDO Y MIGRACIONES: HACIA UNA NUEVA MIRADA GLOBAL}

\subsection{Los estudios migratorios en el Estructuralismo}

Aunque en la mayoría de publicaciones sobre teorías migratorias suela figurar como una de las grandes corrientes de estudio (Massey et al. 1998; King 2012), lo cierto es que el Estructuralismo ha sido pobremente explotado en la literatura sobre migraciones. $\mathrm{Al}$ margen de unas pocas obras destinadas al análisis de los flujos de población desde la óptica del sistema-mundo (Jones y Mielants 2011; Grosfoguel et al. 2016) y algún artículo (Molinero 2017), lo más común ha sido encontrar autores cuyos análisis dan por buena esta perspectiva sin directamente desarrollarla teóricamente, como ha sido el caso de Sayad (2010) o Sassen (1999).

En el caso de la ecología-mundo la conexión teórica ha sido incluso menor, dada la menor vigencia e impacto del paradigma respecto a su precedente. Tan solo un artículo publicado en la revista Relaciones Internacionales (2016) teorizando sobre el rol estructural que cumplen los migrantes en el sostenimiento de la agricultura capitalista global y el número coordinado por Avallone en la revista Theomai (2018a) sobre migraciones postcoloniales en la agricultura global, han contribuido a desarrollar este nexo teórico.

\subsection{Estudiar las migraciones a través de la ecología-mundo}

La ecología-mundo aporta un enfoque principalmente de tipo historiográfico cuyas categorías analíticas centradas en relaciones socio-ecológicas y no puramente sociales, permiten trascender el ámbito de los estudios migratorios tradicionales. Al conectar la acumulación de capital, la búsqueda del poder y la co-producción de la naturaleza en una unidad dialéctica se constituye como "una manera de pensar a través de la historia humana en la red de la vida" (Patel y Moore 2017: 38) y, por lo tanto, como un paradigma útil para el análisis de los movimientos poblacionales. 
A continuación, se tratará de dar cuenta de la vinculación existente entre las principales categorías utilizadas por el paradigma y los fenómenos migratorios. Se pondrá así en valor tanto el estudio de los movimientos de fronteras de producción, como la relación entre movilidad espacial y trabajo donde la configuración de las áreas de emigración, las relaciones coloniales y la producción de mano de obra migrante barata ocupan un espacio central a nivel sistémico.

\subsection{Fronteras de producción y movimientos poblacionales}

El reconocimiento de las jerarquías socio-ecológicas que regulan las relaciones entre los seres vivos es un pilar del constructo teórico de la ecología-mundo. Estas relaciones asimétricas han sido y son producto de los movimientos de fronteras de producción, cuya expansión ha ido redefiniendo los roles de las naturalezas humanas y extrahumanas dentro del sistema capitalista global. Son así, las actividades de extracción y colonización de nuevas tierras, por parte de los diversos agentes del sistema (especialmente empresas transnacionales y Estados), las cuáles han sostenido directamente la reproducción de las relaciones socioecológicas capitalistas, organizando ambas naturalezas con el fin de garantizar de forma gratuita o barata la obtención de los principales factores de producción.

Los movimientos de frontera son, por lo tanto, fundamentales para entender la movilidad espacial, dado que la conquista de nuevas tierras, la extracción de nuevos minerales o la apropiación de nuevos recursos naturales, han implicado desplazamientos y movilidades poblacionales. Estos movimientos son inherentes a la propia lógica del sistema, pues el capital persigue circular de forma constante, sobrepasando así de forma continua los límites de producción (Marx 1971; Hopkins 1987).

"El capitalismo no sólo tiene fronteras; en un sentido fundamental está definido por el movimiento de frontera. El propio dinamismo de la producción capitalista es impensable en la ausencia de apropiación de frontera que permitió el flujo de más y más materiales a través de una determinada unidad abstracta de tiempo de trabajo. El carácter auto-expansivo del valor funciona solo bajo las condiciones históricas específicas de expansiones geométricas en el volumen material de producción, cuya composición de valor debe ser recurrentemente reducida. Esto puede ocurrir solamente a través de la ampliación continua de las extensiones geográficas para la apropiación" (Moore 2013: 13).

$\mathrm{Al}$ producir cambios en la estructura y distribución espacial de las poblaciones humanas, los movimientos de fronteras generan movilidades, ya sea de forma directa, desplazando a las poblaciones, como de forma indirecta, impulsando las migraciones. En este sentido hay una relación estricta entre el funcionamiento de la ecología-mundo capitalista y las formas de movilidad espacial de los seres vivos. El capitalismo pues, se funda en la movilidad, dado que la búsqueda de bienes baratos y nuevas fronteras forma parte de su esencia, hecho que 
demuestra cómo, a lo largo de la historia, los propios Estados han ido abriendo o cerrando sus propias fronteras a la entrada de migrantes en función de las necesidades productivas y sociales (Sassen 1999). Esta dinámica, por supuesto, no se ha dado sin enfrentar resistencias, manifestadas de diversas formas, incluidas las migraciones. Ello no es un aspecto menor, pues dichas dinámicas, al unirse junto con otras "naturalezas rebeldes, desde las enfermedades hasta las revueltas de los esclavos y campesinos" (Moore 2017b: 299), han tenido una innegable influencia en la construcción del mundo.

La movilidad global de poblaciones determinada por los movimientos de fronteras permite entender los factores impulsores de las migraciones humanas desde una perspectiva sistémica, como parte de las relaciones socio-ecológicas reproducidas por la ecología-mundo. Desde esta óptica, pueden así entenderse los determinantes de numerosas migraciones masivas tanto históricas como actuales. Claros ejemplos de ello serían tanto la hambruna irlandesa de la papa del 1845-1851, que provocó la emigración de más de un millón de personas en pocos años, como los cambios en la agricultura del 1800, que generaron millones de emigrantes en Europa (Bauman 2011). En la actualidad, desplazamientos poblacionales como los producidos por el cambio climático o la fuga de las áreas donde ha regresado la malaria, especialmente en la periferia global, pueden claramente ser explicados como producto de la articulación de la ecología-mundo moderna. Numerosas investigaciones han reconocido la conexión existente entre deforestación y calentamiento global, por un lado, y la difusión de la malaria, por el otro, activando una retroacción sobre los seres humanos, que se han visto forzados a abandonar las áreas afectadas por estos procesos (Olson, Gangnon, Silveira y Patz 2010), evidenciando de esta manera "la complejidad de las relaciones entre el uso de la tierra, el fenómeno climático, la diversidad de las especies y la transmisión de las enfermedades" (Myers y Patz, 2009, 236).

Un elemento que el análisis de la ecología-mundo tiende a recalcar como determinante en estos procesos es también el carácter colonial que la búsqueda, conquista y apertura de nuevas fronteras conlleva. La historia del azúcar, por ejemplo, da cuenta de cómo este alimento ha ocupado un espacio central en la construcción de la ecología-mundo, al constituirse en frontera apropiada mediante la transformación de territorios para el monocultivo, cuya explotación requirió del desplazamiento y sumisión de poblaciones para el trabajo esclavo (Moore 2000). Estos procesos de conquista territorial, por lo tanto, implicaron movilidades forzosas, pero también migraciones de quiénes residían en esos territorios. En este sentido, la apropiación de fronteras produce tanto fugas como desposesiones. Esto no es una mera casualidad o el producto de contextos específicos, se trata del éxito de una violencia ejercida por determinados actores políticos y sociales para garantizar el avance del proceso de acumulación. La movilidad espacial de la población humana, con diferentes grados de coacción, es una necesidad de la ecología-mundo capitalista, por lo tanto, un éxito y una condición constitutiva de su manera de funcionar. 


\subsection{Centralidad de las áreas de emigración y relaciones post-coloniales}

Al comprender la centralidad que el concepto de frontera de producción ocupa en el estudio de la ecología-mundo y su estricta relación con la movilidad espacial, humana y extra-humana, se puede entender la relevancia de adoptar una perspectiva holística del fenómeno migratorio para este análisis. Como ya evidenció Sayad (2010), el proceso migratorio requiere ser estudiado en su conjunto, no solo prestando atención al momento de la inmigración (destino), sino también al de la emigración (origen), algo que la ecología-mundo, gracias a su enfoque transnacional, permite conectar en una única unidad de análisis.

Como el enfoque teórico de Sayad, el de la ecología-mundo sitúa el estudio de las relaciones migratorias en el contexto histórico de las relaciones de poder a nivel global. Esto significa asumir que las relaciones migratorias solo se pueden entender si se tienen en cuenta las jerarquías asimétricas entre áreas geográficas cuyas relaciones geo-políticas y geo-económicas relativas determinan las distintas posiciones que cada Estado (y por ende sus poblaciones) ocupan en la división internacional del trabajo.

La posición que los Estados ocupan en la ecología-mundo marcará no solo el estatus jerárquico de éstos en la gobernanza global, sino también el de sus migrantes, que portarán ese peso simbólico en los lugares de destino. "En efecto, entre las características que conducen a constituir la inmigración en sistema figuran, y en primer lugar, las relaciones de dominación que prevalecen a escala internacional" (Sayad 2010: 234).

Las relaciones de fuerza entre Estados y economías son, por lo tanto, transmitidas a los actores del proceso migratorio, reproduciendo las jerarquías globales de poder también al nivel "de la lógica del orden simbólico" (Sayad 2008: 38), expresión de la "relación de dominante a dominado, objetivamente inscrita en la relación de país de inmigración a país de emigración" (Sayad 2010: 126).

De esta forma se entiende que las migraciones se refieren a relaciones entre poblaciones con estatus sociales y posiciones de poder asimétricas cuya herencia proviene de los procesos coloniales. Unos hechos que no fueron solo conquistas espaciales, sino que tuvieron una ideología reflejada en el principio de colonialidad, es decir, que se basaban en una organización jerárquica de las relaciones sociales, culturales y epistémicas entre los diferentes pueblos. Así, como señalaba Aníbal Quijano (2000: 342), se caracterizaron por "la imposición de una clasificación racial/étnica de la población del mundo".

La centralidad de las fronteras de producción destaca por lo tanto la relevancia de las relaciones (post) coloniales y, por ende, de las construcciones ideológicas, simbólicas y filosóficas que han legitimado y siguen legitimando históricamente las jerarquías a nivel global. Las ideas y construcciones simbólicas son determinantes y funcionan como fuerzas materiales (Moore 2016b). Entre estas ideas, el análisis de la ecología-mundo resalta las del racismo y racialización de las poblaciones, conectando determinantes históricos con relaciones simbólicas contemporáneas. En su análisis del Capitaloceno, Moore reconoce que hablar de éste en lugar de Antropoceno, permite ver el capitalismo como una manera de 
organizar las relaciones entre los seres humanos y el resto de la naturaleza en la que "muchos de los sucesos que ocurren en la era capitalista no son llevados a cabo por los capitalistas: son manejados por los Estados, y están habilitados por culturas de la dominación, especialmente el racismo y el sexismo" (Velednitski 2017). Así pues, racismo y procesos de racialización contribuyen a la producción y reproducción de las naturalezas baratas (Moore 2015a) y, por lo tanto, a la división de la población humana mundial en áreas distintas con derechos diferenciados y jerarquizados, útiles para el proceso de acumulación. Los migrantes, gobernados por los Estados de destino desde el racismo (Sayad 2010), se insertan así en una lógica global de la discriminación donde las poblaciones racializadas son consideradas como parte de la naturaleza:

"La idea que los seres humanos están fuera de la naturaleza tiene una historia larga. Es una construcción del mundo moderno (...) Pero la mayoría de los humanos fueron incluidos en la categoría de "Naturaleza", la cual era percibida como algo que debía ser controlado, dominado y puesto a trabajar y civilizar "(Moore y Ahsan 2015).

El dualismo jerárquico naturaleza/sociedad es parte de un conjunto más amplio de dualismos que separan y jerarquizan las poblaciones (humanas y extra-humanas) - blancos y negros, civilizados y no civilizados, nacionales y no nacionales, hombres y mujeres, colonizadores y colonizados - permitiendo la producción de naturalezas baratas y legitimando los movimientos de las fronteras de producción y sus apropiaciones como explica Moore (2017: 242):

"En este movimiento, la naturaleza barata encarna una lógica de abaratamiento en un sentido ético-político, reubicando numerosos -a veces la mayoría- humanos en la naturaleza, lo mejor para que su trabajo sea no remunerado, devaluado e invisibilizado.'

En este sentido, se puede hablar de la construcción, a lo largo de la modernidad, de una colonialidad del trabajo (Avallone 2018b), caracterizada por el hecho de que cuanto mayor sea el acercamiento del trabajo a la reproducción social y a la naturaleza, menor será su reconocimiento social. De esta manera, se determina una jerarquía de los diferentes trabajos en relación a su distancia con la reproducción social: el valor del trabajo, y de las poblaciones que lo realizan, se reduce a cero cuando es totalmente de reproducción, como en el caso del trabajo doméstico sin valor económico o los trabajos reservados a los migrantes (especialmente el trabajo agrícola y los cuidados), y crece cuanto más se acerca al mundo de la producción, es decir del valor de cambio.

La construcción racializada de colectivos de poblaciones dominadas ha sido, pues, constitutiva de la ecología-mundo capitalista, favoreciendo la apropiación barata de diversas naturalezas y, con ella, la producción barata de la naturaleza extra-humana. Un caso central en el relato de Moore es la historia de Potosí, un ejemplo histórico, que destaca el nexo entre dominación de la naturaleza (humana y extra-humana) y definición de los "otros" como inferiores, por ser de raza diferente (Moore 2010). En este sentido, Vergés (2017) habla de "Capitaloceno racial", reconociendo la centralidad que tanto el colonialismo, como la racia- 
lización y la esclavitud han ocupado en la constitución de la ecología-mundo capitalista desde sus orígenes.

\subsection{Produciendo mano de obra migrante barata}

El enfoque de la ecología-mundo reconoce que el capitalismo se sostiene mediante la búsqueda y producción constantes de factores de producción baratos. Un proceso que, históricamente, ha sido posible a través de la movilización y apropiación de las naturalezas. Sin embargo, estas en sí mismas no tienen valor, ya sea barato u oneroso, se trata por lo tanto de una subordinación fruto de una construcción social y política útil para el sistema.

El proceso de "valorización" se articula mediante el empleo de diversas herramientas. En el caso del trabajo, una importante parte de la mano de obra barata es producida por parte del Estado, mediante las fronteras, a través del manejo de la movilidad humana espacial (Sassen, 1999). Los migrantes, por lo tanto, no son trabajadores vulnerables en sí mismos, sino que son producidos como mano de obra debilitada mediante leyes y políticas estatales e internacionales. Ejemplos de ello son la reducción, el cierre o el incremento de la selectividad a las posibilidades de movilidad e ingreso legal en los Estados. En este sentido Moore afirma que:

"La historia del capitalismo se caracteriza por la búsqueda de trabajo barato (como parte de la naturaleza barata) y la construcción de las fronteras está diseñada para facilitar el abaratamiento. Las fronteras son generalmente presentadas como barreras a los trabajadores, lo cual es cierto. Pero están construidas y vigiladas para aterrorizar, cultural y físicamente, a los trabajadores y por ende para abaratar sus vidas y trabajo" (entrevista realizada por los autores a Jason W. Moore el 2 de septiembre de 2018).

El migrante es un cuerpo extraño que penetra al Estado nación pero que "sólo existe por el trabajo, para el trabajo, en el trabajo; fuera del trabajo no hay salvación para él" (Sayad 2010a: 252), un trabajo barato indispensable para el mantenimiento del sistema. La ecología-mundo permite así entender el fenómeno migratorio desde una perspectiva holística y sistémica al plantear un paradigma de análisis global que tiene en cuenta tanto las áreas de origen como las de destino. A la par, permite explicar tanto el rol jerárquico que ambas cumplen en la estructura capitalista global como los factores estructurales que hacen que este proceso no solo acontezca, sino que se reproduzca de forma constante a lo largo de la historia. 


\section{CONCLUSIONES}

El marco de la ecología-mundo es versátil, interdisciplinario y permite tanto amparar numerosos desarrollos teóricos como testar infinitas hipótesis sobre las dinámicas intervinientes en los procesos migratorios.

Las migraciones, en tanto que fenómenos transnacionales y multiespaciales, requieren una superación del marco estato-etno-céntrico para ser entendidas como hechos sociales totales (Sayad 2010) siendo este paradigma una perfecta herramienta para ello. La ecología-mundo no solo es una teoría de lo internacional, sino que además propone un análisis geo-histórico y sistémico cuya propuesta permite abarcar el estudio de todo movimiento poblacional en base a su función y resistencia dentro de la estructura capitalista global.

Fenómenos como el trabajo migrante agrícola, observables como tendencia creciente a nivel global, pueden entenderse mediante la estrategia del sistema para garantizar la producción de comida barata, reduciendo a su vez el coste del trabajo global (Molinero y Avallone 2016). Pero otras migraciones, como las llamadas "ambientales" también pueden entenderse como productos de las dinámicas constitutivas de la ecología-mundo al ser movilidades producidas por la apropiación de la naturaleza (Felli 2016).

Las posibilidades de interconexión entre el paradigma analizado y los estudios migratorios son infinitas, siendo un campo de estudio aún por explorar en profundidad. Este artículo ha querido por un lado presentar la teoría de la ecología-mundo con el objetivo de dar a conocer sus postulados a un público más amplio, y, por el otro, presentar algunas de las líneas teóricas que permiten conectar sus fundamentos con los estudios sobre migraciones. El campo por explorar entre migraciones y ecología-mundo es inmenso, ofreciéndose una amplia ventana al mundo de la investigación para producir en los próximos tiempos estudios innovadores que permitan entender mejor los determinantes y procesos históricos o actuales de la movilidad humana.

\section{BIBLIOGRAFÍA}

ARRIGHI, G. (2002): "Global Capitalism and the Persistence of the North-South Divide", Science \& Society, 65 (4), pp. 469-76.

ARRIGHI, G. y Moore, J.W. (2001): "Capitalist Development in World-Historical Perspective" en Phases of Capitalist Development. Booms, Crises and Globalizations, Nueva York, Palgrave, pp. 56-75.

AVALLONE, G. (2018a): "Trabajo y Migraciones Postcoloniales En La Agricultura Capitalista Global", Theomai, 38, pp. 5-7.

AVALLONE, G. (2018b): "Migraciones postcoloniales, agricultura global y colonialidad del trabajo", Theomai, 38, pp. 91-102.

BAIR, J. (2009): "Global Commodity Chains. Genealogy and Review." en Frontiers of Commodity Chain Research, Redwood, Stanford University Press, pp. 1-34.

BAUMAN, Z. (2011): Memorias de clase. La prehistoria y la sobrevida de las clases, Buenos Aires, Nueva Visión. 
BRAUDEL, F. (1976): El Mediterráneo y El Mundo Mediterráneo En La Época de Felipe II, Ciudad de México, Fondo de Cultura Económica.

BROWN, C. y AINLEY, K. (2009): Understanding International Relations. Basingstoke y New York, Palgrave Macmillan.

CALDENTEY, E., SUNKEL, O. y TORRES, M. (2012): Raúl Prebisch (1901-1986): Un Recorrido Por Las Etapas de Su Pensamiento Sobre El Desarrollo Económico, Santiago de Chile, CEPAL.

DE CASTRO, C. (2018): "Disputando la frontera. Las dimensiones políticas en las redes globales de producción y en el desarrollo regional y local", Ager, 24, pp. 9-34.

FELLI, R. (2016): "Les migrants climatiques: de la menace sécuritaire à l'instrumentalisation entrepreneuriale" en La Grande Adaptation. Climat, capitalisme et catastrophe, Paris, Le Seuil.

GROSFOGUEL, R., MALDONADO-TORRES, N. y SÁLDIVAR, J.D., (2016): No Latin@s in the World-System: Decolonization Struggles in the Twenty First Century U.S. Empire, Abingdon y New York, Routledge.

HOPKINS, T.K. y WALLERSTEIN, I. (1986): "Commodity Chains in the WorldEconomy Prior to 1800", Review, 10 (1), pp. 157-70.

JONES, T. y MIELANTS, E. (2011): Mass Migration in the World-System: Past, Present and Future, Boulder, Paradigm Publishers.

KING, R. (2012): "Theories and Typologies of Migration: An Overview and a Primer", Willy Brandt Series of Working Papers in International Migration and Ethnic Relations, 3/12, Malmö.

MASSEY, D.S., ARANGO, J., HUGO, G., KOUAOUCI, A. y PELlEGRINO, A. (1998): Worlds in Motion: Understanding International Migration at the End of the Millennium, Oxford, Clarendon Press.

MEZZADRA, S. y NEILSON, B. (2017): La frontera como método, Madrid, Traficantes de sueños.

MOLINERO, Y. (2018): "Ejerciendo Agencia En Las Cadenas Agrícolas Globales. Del Modelo Boliviano En La Agricultura Argentina a La Situación de Los Migrantes Marroquíes En La Agricultura de La Piana Del Sele (Salerno, Italia)", Theomai, 38, pp. 68-90.

MOLINERO, Y. (2017): "Mass Migrations across the World-System's History." E-International Relations, disponible en https://www.e-ir.info/2017/08/01/mass-migrations-across-the-world-systems-history/ [consulta: 27-08-2018]

MOLINERO, Y. y AVALLONE, G. (2016): "Produciendo Comida y Trabajo Baratos: Migraciones y Agricultura En La Ecología-Mundo Capitalista”, Relaciones Internacionales, 33, pp. 31-51.

MOORE, J.W. (2017a): "World Accumulation and Planetary Life, or, Why Capitalism Will Not Survive until the 'Last Tree Is Cut'”, IPPR Progressive Review, 24 (3), pp. 176-202.

MOORE, J.W. (2017b): "Metabolic Rift or Metabolic Shift? Dialectics, Nature, and the World-Historical Method", Theory and Society, 46 (4), pp. 285-318.

MOORE, J.W. (2017c): "The Capitalocene Part II: accumulation by appropriation and the centrality of unpaid work/energy", The Journal of Peasant Studies, 45 (2), pp. 237-279.

MOORE, J.W. (2016a): Anthropocene or Capitalocene? Nature, History, and the Crisis of Capitalism, Oakland, PM Press. 
MOORE, J.W. (2016b): "El Fin de La Naturaleza Barata: O Cómo Aprendí a Dejar de Preocuparme Por 'El' Medioambiente y Amar La Crisis Del Capitalismo", Relaciones Internacionales, 33, pp. 143-74.

MOORE, J.W. (2015a): Capitalism in the Web of Life: Ecology and the Accumulation of Capital, Nueva York, Verso.

MOORE, J.W. (2015b): Ecologia-Mondo e Crisi Del Capitalismo. Ombre Corte, Verona, Ombre Corte.

MOORE, J.W. y AHSAN, K. (2015): Capitalism in the Web of Life: An Interview with Jason W. Moore, disponible en

https://www.viewpointmag.com/2015/09/28/capitalism-in-the-web-of-life-an-interviewwith-jason-moore/ [consulta 17-10-2018]

MOORE, J.W. (2013): "El Auge de La Ecología-Mundo Capitalista (I) Las Fronteras Mercantiles En El Auge y Decadencia de La Apropiación Máxima”, Laberinto, 38, pp. 9-26.

MOORE, J.W. (2010): "This lofty mountain of silver could conquer the whole world": Potosí and the political ecology of underdevelopment, 1545-1800", The Journal of Philosophical Economics, IV(1), pp. 58-103

MOORE, J.W. (2003): "Capitalism as World-Ecology: Braudel and Marx on Environmental History", Organization \& Environment, 16 (4), pp. 431-58.

MOORE, J.W. (2000): "Sugar and the Expansion of the Early Modern World-Economy", Review, 23(3), pp. 409-433.

MYERS, S.S. y PATZ, J.A. (2009): "Emerging Threats to Human Health from Global Environmental Change", Annual Review of Environment and Resources, 34, pp. 223-252.

OLSON S.H., GANGNON R., SILVEIRA G.A. y PATZ, J..A. (2010): “Deforestation and Malaria in Mancio Lima County, Brazil", Emerging Infectious Diseases, 16, pp. 1108-1115.

PATEL, R. y MOORE, J.W. (2017): A History of the World in Seven Cheap Things. A Guide to Capitalism, Nature and the Future of the Planet, Oakland, University of California Press.

QUIJANO, A. (2000): “Colonialidad del poder y clasificación social”, Journal of WorldSystems Research, VI (2), pp. 342-386.

SASSEN, S. (1999): Guests and Aliens, Nueva York, The New Press.

SAYAD, A. (2010): "Elementos Para Una Sociología de La Inmigración”, Empiria, 19, pp. 251-57.

SAYAD, A. (2008): "Estado, nación e inmigración. El orden nacional ante el desafío de la inmigración", Apuntes de Investigación del CECYP, 13, pp. 101-116.

STREET, J.H. y DILMUS, J.D. (1980): "Institucionalismo, Estructuralismo y Dependencia En América Latina", Comercio Exterior, 32 (12), pp. 1297-1303.

VELEDNITSKY, S. (2017): "The Case for Ecological Reparations: A Conversation with Jason W. Moore", disponible en http://edgeeffects.net/jason-w-moore/ [consulta 1810-2018]

WALLERSTEIN, I. (2000): “World-Systems Analysis.” en The Essential Wallerstein, Nueva York, The New Press, pp. 129-148

WALLERSTEIN, I. (2004): “The Modern World-System as a Capitalist World-Economy: Production, Surplus Value, and Polarization" en World-Systems Analysis. An Introduction, Durham, Duke University Press, pp. 23-41.

WALLERSTEIN, I. (2000): The Essential Wallerstein, Nueva York, The New Press 
WALLERSTEIN, I. (1974): The Modern World-System I: Capitalist Agriculture and the Origins of the European World-Economy in the Sixteenth Century, Nueva York y Londres, Academic Press.

WARREN, B. (1980): Imperialism: Pioneer of Capitalism, Londres, Verso. 